\title{
Role of Clusterin in the Female Reproductive System
}

\author{
Eliza Shrestha, Yang Yue-bo, Li Xiao-Mao \\ Dept of Gyn, The Third Affiliated Hospital of Sun Yat-sen University, \\ Guangzhou 510630, China
}

\begin{abstract}
Clusterin is a glycoprotein that was initially isolated from the male reproductive system. Subsequently, clusterin has been found to be widely distributed in a variety of tissues in mammals. One characteristic of the expression of clusterin is that it is induced as a result of cellular injury, death or pathology. Despite the efforts of many laboratories working in diverse biological systems, the function of clusterin remains unknown. Recent studies show that it has strong relationship with gynecological malignancies. Overall, the evidence suggests that function of clusterin is to protect surviving cells after damage. This protection may result from a detergent-like action of the protein.
\end{abstract}

Keywords: Clusterin; Female reproductive system; chaperone.

\section{Introduction}

Clusterin is glycoprotein first isolated from ram rete testis fluid by Blaschuk, Burdzy, and Fritz in 1983 and so named because of its ability to elicit clustering of sertoli cells. Since that time species Clusterin is an enigma. In spite of having been discovered almost two decades ago ${ }^{[1]}$ as a protein causing clustering of red blood cells hence the name now most widely used clarification of its biological roles has proved exceptionally difficult, and recent studies using clusterin knockout mice 2,3 may have deepened the mystery. The multiplicity of names for clusterin is an indication of the complexity of the problem (Table 1) and could reflect on the one hand its multi functionality or alternatively could mask commonality of function.

\section{Structure of clusterin}

Clusterin is a secreted hetero dimeric glycoprotein of $75-80 \mathrm{kDa}$. A single-copy gene in humans of nine exons, spanning over 16kband located on chromosome $8 \mathrm{p} 21$-p 12 , encodes an mRNA of approximately $2 \mathrm{~kb}$ which directs the synthesis of a 449-amino acid primary polypeptide chain. Proteolytic processing removes the 22-mer secretory-signal polypeptide and cleaves the remainder into $\alpha$ and $\beta$ chains (Fig. 1). These are linked in anti-parallel by five disulfide bridges near their centers, forming a molecule in which the core is flanked by three amphipathic $\alpha$ - helices and two coiled- coil alpha helices. Some $30 \%$ of the mass of the mature protein is $\mathrm{N}$ - linked carbohydrate of variable structure attached at six glycosylation sites. A nuclear localization signal and a potential dinucleotide binding site have also been identified. Clusterin is highly conserved across species, showing $70-80 \%$ identity at the amino acid level among mammals, and numerous variants and isoforms have been described though their functional significances are unclear ${ }^{4,5}$. No $\mathrm{X}$ ray crystallographic data have yet been generated for clusterin, since the protein has proved difficult to crystallize due to its inherent "stickiness".

\section{Biological function}

Unraveling the biological significance of clusterin is complicated by two main characteristics: (1) the propensity of the protein to interact with a wide range of molecules, including itself, lipids, amyloid proteins, components of the complement membrane attack complex, and immunoglobulins; and (2) the ubiquitous upregulated expression of the gene in e.g. developmental remodeling, apoptotic disease

Correspondence

Dr. Eliza Shrestha

Dept of Gyn,

The Third Affiliated Hospital of Sun Yat-Sen University

Guangzhou 510630, China

Mobile : +86 13250526294

E-mail: shresthaeliza@gmail.com 
states such as neurodegeneration, and in response to injury and other stresses (Fig. 1). Clusterin variants additionally may play tissue or context specific roles. Is clusterin therefore truly multifunctional, or can a primary role be extracted from the diversity? Earlier hypotheses focused on specific potential roles including cytoprotection at fluid-tissue boundaries, membrane recycling during development and in response to injury, and regulation of complement-mediated membrane attack. More recently, arguments have been advanced proposing clusterin as a form of secreted heat-shock protein or chaperone molecule, in light of its induced expression by thermal, oxidative or mechanical stress, its diverse ligands, its ability to inhibit stress-induced protein precipitation, and its reported activities in vitro systems as a protectant against cytotoxic agents ${ }^{6,7}$. In the context of active cell death, these lines of evidence argue against a pro-apoptotic role for clusterin, further supported by failure of targeted overexpression of the gene to cause increased death of photoreceptors in a transgenic model $^{8}$. The posited role of clusterin as a cytoprotective chaperone-like molecule therefore seems compelling. However, not all forms of clusterin exhibit these benign properties. Recently, a truncated $55 \mathrm{kDa}$ form of clusterin induced by irradiation in vitro has been found targeted to the nucleus where it appears to act as a death signal ${ }^{9}$. Interestingly, investigations of knockout mice have also highlighted the two faces of clusterin, since on the one hand absence of clusterin reduces cell death in hypoxia-ischemia-induced brain damage, suggesting that it normally functions to exacerbate neuronal damage in these circumstances ${ }^{2}$, whereas in the same knockout strain, autoimmune myocardial damage is increased, implying a normally protective role ${ }^{3}$. It is possible, therefore, that as with a select few other molecules such as p53 or Bcl-x, clusterin can act either to promote or to inhibit cell death, depending on the cellular context or molecular species.

\section{Synthesis and degradation}

Clusterin is constitutively expressed in almost all mammalian tissues, and is a major protein in physiological fluids including plasma, milk, urine, cerebrospinal fluid and semen. Within particular given tissue, clusterin may be expressed predominantly in particular cell types for example, in epithelial cells at tissue fluid interfaces or in specific sub-populations of neurons. Clusterin mRNA is translated on membranebound ribosomes and the polypeptide undergoes processing from initial removal of the signal sequence in the endoplasmic reticulum, through a mannose rich 58-65 kDa form, to cleavage, dimer assembly and final glycosylation steps in the trans Golgi network. Secretion occurs from secretory vesicles or through non-regulated routes, and uptake and degradation may be mediated by the endocytic receptor gp330/megalin
(LRP-2), a member of the low-density lipoprotein receptor gene family ${ }^{10}$. Truncated clusterin forms, potentially derived from alternative translational start codons, may escape glycosylation and be targeted to the nucleus ${ }^{5}$. Up regulation of clusterin mRNA and protein are widespread phenomena in developmental and pathophysiological states, suggesting that control of expression levels is important. Analysis of the promoter has revealed numerous transcription factor-binding sites ${ }^{11}$ but also a conserved "clusterin element" CLE, which appears related to heat-shock element consensus sequences, can bind the HSF-1 transcription factor, and may govern stress-response induction ${ }^{6}$. Factors such as TGF have also been implicated in modulating clusterin transcription ${ }^{5}$.

\section{Impact of clusterin in different gynecologic malignancies}

\section{In human ovarian carcinoma}

Ovarian carcinoma is a major cause of death among all gynecolgoic malignancies. It is the seventh most common malignancy in women worldwide ${ }^{12}$. Recently, the incidence of ovarian carcinoma has been increasing in Asian Countries ${ }^{13}$. The overall 5 year survival rate is only approximately $30 \%$, and this poor prognosis usually is ascribed to late tumor diagnosis ${ }^{14}$. The development of ovarian carcinoma is considered a multistep process that involves multiple genetic changes ${ }^{15}$. Therefore genes that are expressed differentially in ovarian carcinoma were investigated by serial analysis of gene expression (SAGE) and quantitative real time reverse transcriptase polymerase chain reaction analysis and overexpression of clusterin gene was detected ${ }^{16}$. Likewise, another study suggested that the clusterin over expression significantly correlates with decresed survival in ovarian cancer patients, and clusterin confers resistance to paclitaxel-induced apoptosis in ovarian cancer cells ${ }^{17}$. The compared expression profiles of primary tumor tissue from five long- term ( $>$ 8years) and five short- term $(<2$ years) ovarian cancer survivors and indentified clusterin as one of the genes that were significantly up-regulated in short term survivors. Still the potential role in the development and progression of ovarian carcinoma is unclear. Up regulated expression of cytoplasmic clusterin in human ovarian carcinoma suggested that the overexpression of cytoplasmic clusterin may represent an acquired malignant phenotypic feature of ovarian carcinoma and may be one of the important factors in determining aggressive nature of ovarian carcinoma ${ }^{18}$. So it shows that clusterin is strongly associated with ovarian carcinoma.

\section{In cervical cancer}

Cervical cancer is the seventh most common cancer, overall, and the third most common in women, in 
whom it comprises $9.8 \%$ of all cancers. Generally, it is much more common is developing countries, where $78 \%$ of cases occur and where cervical cancer accounts for $15 \%$ of female cancers, with a life time risk of about $3 \%$, whereas in developed countries it accounts for only $4.4 \%$ of new cancers, with a life time risk of $1.1 \%$.

Cervical carcinoma is generally treated by surgery, radiotherapy or both. The data regarding clusterin expression in cervical cancer identification are very premature; however, there were two studies done recently in cervical cancer.

A series of 52 patients who underwent radical hysterectomy and systematic lymphadenectomy clusterin protein was present in the cytoplasm of cervical cancer cells ${ }^{19}$. The expression of clusterin protein in invasive cervical cancer tissues was not related to any clinicopathologic factors analyzed. Patients with positive clusterin expression showed significantly worse prognosis than those with negative clusterin expression $(\mathrm{p}=0.017)$. Multivariate analysis including clusterin expression revealed that clusterin expression $(p=0.006)$ and the number of positive node groups $(\mathrm{p}=0.002)$ were independent prognostic factors for survival. Survival of patients with invasive cervical cancer could be stratified into three groups by combination of clusterin expression and number of positive node groups with an estimated 5-year survival rate of $100.0 \%$ for no or one positive node group irrespective of clusterin expression (group A), $78.7 \%$ for multiple node groups with negative clusterin expression (group B), and $14.3 \%$ for multiple node groups with positive clusterin expression (group C) ( $\mathrm{p}=0.03$ for group $\mathrm{A}$ vs. group $\mathrm{B}, \mathrm{p}=0.004$ for group $\mathrm{B}$ vs. group $\mathrm{C}$, and $\mathrm{p}=0.0001$ for group $\mathrm{A}$ vs. group $\mathrm{C}$ ). So Clusterin expression and the number of positive node groups were independent prognostic factors for invasive cervical cancer patients treated with radical hysterectomy and systematic lymphadenectomy. Clusterin might be a new molecular marker to predict the survival of cervical cancer patients with multiple positive node groups. Another recent study was Clusterin confers paclitaxel resistance in cervical cancer shown that clusterin expression was significantly higher in cervical cancer tumor samples than in normal cervical tissues and that clusterin expression was significantly correlated with paclitaxel resistance in cervical cell lines ${ }^{20}$. The result suggests that Cervical cancer tissues expressed significantly higher levels of clusterin than did normal cervical tissues (4.08 vs. $1.35, \mathrm{P}<0.05)$. Clusterin expression levels were correlated with paclitaxel resistance in cervical cancer cell lines, and transfection of clusterin
siRNA into HeLaS3 cells significantly decreased their resistance to paclitaxel $(\mathrm{P}<0.05)$.

\section{In endometrial cancer}

Endometrial cancer is the commonest gynaecological cancer mostly affecting women in the postmenopausal age group. It ranks fourth in terms of incident cancers in women, and eighth in terms of age-adjusted mortality ${ }^{21}$. Several risk factors are known to be associated with endometrial cancer, the most significant being unopposed oestrogens.

Endometrial carcinoma is divied into endometriod and papillary serous type carcinoma according to the histological characteristics. As we know clusterin has been associated with various malignacies. A higher expression of clusterin were found in endometrioid compared to papillary serous carcinoma using both immunohistochemistry $(\mathrm{p}=0.033)$ and Western blot analysis $(\mathrm{p}=0.024)$. The mRNA and protein expressions of clusterin in endometrioid carcinoma were higher than in benign endometrium $(\mathrm{p}=0.002)$. Forced expression of clusterin promoted 293T cell survival in a concentration-dependent manner, and estradiol treatment increased clusterin expression in HEC1B but not in HEC-1A cells. So these data suggest that clusterin expression is realted to endometrioid carcinoma of endometrium, in which estrogen is involved in the regulatory network of clusterin ${ }^{22}$. Eighty percent of endometrial carcinoma is the endometrioid type. In contrast, uterine papillary serous carcinomas account for only $3 \%$ of cases. This type usually recurs and metastasizes distantly even after the surgical treatment of carcinoma at an early stage ${ }^{23,24}$. This study results suggest that sCLU is an important factor that can distinguish the two different types of carcinogenesis. A previous study in endometrium showed that sCLU in endometrium is associated with tissue remodeling by mediating clearance or neutralizing the tissue debris at the late secretory phase when the endometrium undergoes dynamic growth and involution ${ }^{25}$. As endometrium is a dynamic organ that undergoes regular cycles of remodeling under hormonal control ${ }^{26-28}$.

\section{Conclusion}

Either clusterin is a multi-functional protein, or its appearance in various tissues and its association with various processes has some fundamental link. It is now very clear that the clusterin is made primarily by the surviving cells or the cells next to the dying cells ${ }^{14,15}$. Despite the efforts of many laboratories working in diverse biological systems, the function of clusterin remains unknown. Overrall, the evidence suggests that function of clusterin is to protect surviving cells after damage. 


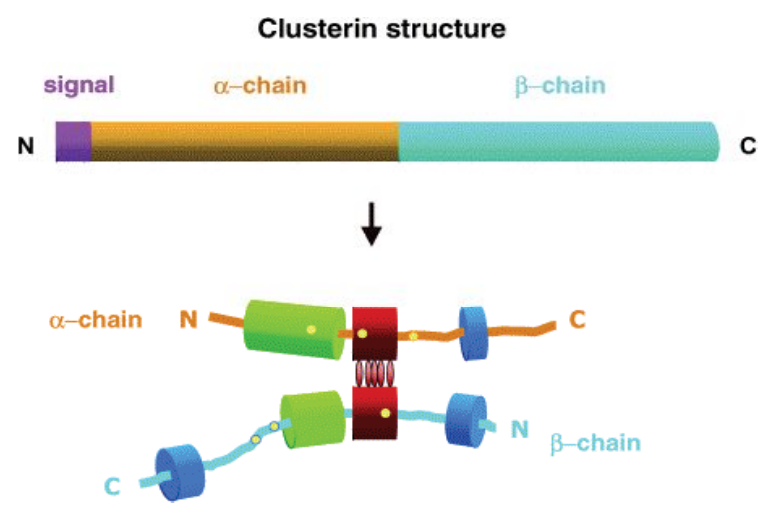

Fig 1. Schematic representation of the structure of human clusterin. The precursor polypeptide chain (top) is cleaved proteolytically to remove the 22 -mer secretory signal peptide (magenta) and subsequently between residues 227/228 to generate the alpha (orange) and beta (light blue)-chains. These are assembled in anti-parallel to give a heterodimeric molecule (bottom) in which the cysteine-rich centers (red) are linked by five disulfide bridges (red ellipses) and are flanked by two predicted coiled-coil alpha helices (green) and three predicted amphipathic alpha helices (dark blue). The six sites of $\mathrm{N}$ linked glycosylation are indicated as yellow spots.

Table 1. A selection of the alternative names by which clusterin is also known, with the species and biological associations which led to their first identification

\begin{tabular}{lll}
\hline Name & Species & Association \\
\hline Clusterin & Sheep & Seminal fluid protein causing cell aggregation \\
SGP-2 & Rat & Sulfated glycoprotein secreted by Sertoli cells of the testis \\
SP-40, 40 & Human & Plasma component, present in SC5b-9 complement complex \\
PADHC-9 & Human & Up regulated in Alzheimer disease brain \\
TRPM-2 & Rat & Up regulated mRNA in prostate following castration \\
CLI & Human & Inhibitor of complement-mediated cell lysis \\
T64 & Quail & Induced in neuroretina \\
GP III & Bovine & Present in chromaffin granules \\
ApoJ & Human & Serum protein with apolipoprotein properties \\
XIP8 & Human & Truncated nuclear form in radiation induced cell death \\
\hline
\end{tabular}

\section{References}

1. IB Fritz, K Burdzy, B Setchell, O Blaschuk, Ram. Rete testis fluid contains a protein (clusterin) which influences cell-cell interactions in vitro, Biol Reprod 1983; 28 (5) :1173-1188.

2. BH Han, RB DeMattos, LL Dugan, JS Kim-Han, RP Brendza, JD Fryer, M Kierson, J Cirrito, K Quick, JAK Harmony, BJ Aronow, DM Holtzman. Clusterin contributes to caspase-3independent brain injury following neonatal hypoxia-ischemia, Nat. Med 2001; 7 (3) : 338-343.

3. L Mc Laughlin, G Zhu, M. Mistry, C Ley-Ebert, WD Stuart, CJ Florio, PA Groen, SA Witt, TR Kimball, DP Witte, JA Harmony, BJ Aronow. Apolipoprotein $\mathrm{J} /$ clusterin limits the severity of murine autoimmune myocarditis, J Clin. Invest 2000; 106 (9) :1105-1113.
4. PWong, BAPfeffer, SL Bernstein, ML Chambers, GJ Chader, ZF Zakeri, Y-Q. Wu, M.R. Wilson, S.P. Becerra. Clusterin protein diversity in the primate eye, Mol. Vis. 6 (2000): 184"C191.

5. KB Reddy, G Jin, MC Karode, JAK Harmony, $\mathrm{PH}$ Howe. Transforming growth factor (TGF)induced nuclear localization of apolipoprotein $\mathrm{J} /$ clusterin in epithelial cells, Biochemistry 35 (19) (1996) 6157"C6163.

6. D Michel, G Chatelain, S North, G Brun. Stressinduce transcription of the clusterin/apoJ gene. Biochem J1997; 328 (1) :45"C50.

7. MR Wilson, SB Easterbrook-Smith. Clusterin is a secrete mammalian chaperone, Trends Biochem Sci. 200025 (3) : 95-98.

8. C Jomary, G Chatelain, D Michel, A Weston, MJ Neal, SE Jones. Effect of targeted expression of clusterin in photoreceptor cells on retinal 
development and differentiation, J Cell Sci. 1999112 (10) : $1455^{\circ} \mathrm{C} 1464$.

9. CR Yang, K Leskov, K Hosley-Eberlein, T Criswell, JJ Pink, TJ Kinsella, DA Boothman. Nuclear clusterin/XIP8, an X-ray-induced Ku70binding protein that signals cell death. Proc. Natl. Acad. Sci. 200097 (11) : 5907-5912.

10. MZ Kounnas, EB Loukinova, S Stefansson, JA Harmony, BH Brewer, DK Strickland, WS Argraves. Identification of glycoprotein 330 as an endocytic receptor for apolipoprotein J/clusterin, J. Biol. Chem. 1995270 (22) : 13070-13075.

11. M Tenniswood, Z Wang, J Lakins, C Morrissey, J O'Sullivan, H Tang. Clusterin in the male reproductive tract, J. Androl. 1998191 (5) : 508-516.

12. PA Wingo, LA Ries, HM Rosenberg, DS Miller, BK Edwards. Cancer incidence and mortality, 1973-1995, a report card for the U.S. Cancer. 1998; 82:197-207.

13. HT Lynch, MJ Casey, J Lynch, TE White, AK Godwin. Genetics and Ovarian carcinoma. Semin Oncol 1998; 25:265-280

14. MS Piver, J Fanning, KA Crag. Cancer of the ovary. In: Knapp RC, RS Berkowitz, editors. Gynecolgic oncology. New York: McGraw-Hill, 1992:250-291.

15. NAuersperg, MI Edelson, SC Mok, SW Johnson, TC Hamilton. The biology of ovarian cancer. Semin Oncol. 1998;25:281-304.

16. CD Hough, CA Sherman-Baust, ES Pizer, et al. Large-scale serial analysis of gene expression reveals genes differentially expressed in ovarian cancer. Cancer Res 2000; 6281-7.

17. CP Dong, GY Seung, YS Eun, C. Mok Samuel, HK Dae. Clusterin confers paclitaxel resistance in Ovarian cancer Neoplasia 2008; 10 : 964 972.

18. D Xie, Sze HL, ST Johathan et al. Upregulated expression of cytoplasmic clusterin in human ovarian carcinoma. Cancer 2005 Jan 15;103(2):277-83

19. H Watari, Yoko Ohta, MK Hassan, Y Xiong,
S Tanaka, N Sakuragi. Clusterin expression predicts survival of invasive cervical cancer patients treated with radical hysterectomy and systematic lymphadenectomy Gynecologic Oncology 108 (2008)527-532.

20. CP Dong, GY Seung, YS Eun, C Mok Samuel, HK Dae. Clusterin confers paclitaxel resistance in cervical cancer. Gynecologic Oncology 2006; 103 : 996-1000.

21. SH Landis, T Murray, S Bolden, PA Wingo. Cancer Statistics, 1999. Cancer Stat 1999; 49:8-31

22. HJ Ahn, J Bae, S Lee, JE Ko, S Yoon, SJ Kim, N Sakuragi. Differential expression of clusterin according to histological type of endometrial carcinoma. Gynecologic Oncology 2008; 110 : 222-229.

23. BA Goff, D Kato, RA Schmidt, M Ek, JA Ferry, HG Muntz, et al. Uterine papillary serous carcinoma: patterns of metastatic spread. Gynecol Oncol 1994; 54:264-8

24. EG Silva, R Jenkins. Serous carcinoma in endometrial polyps. Mod Pathol 1990; 3:120-8.

25. TL Brown, BC Moulton, VV Baker, J Mira, JA Harmony. Expression of apolipoprotein $\mathrm{J}$ in the uterus is associated with tissue remodeling. Biol Reprod 1995; 52:1038-49.

26. R Rugh. The Mouse, Its Reproduction and Development, 2nd ed. Oxford: Oxford Science; 1990: 7-101.

27. CA Finn, DG Porter. The Uterus. Acton, MA: Publishing Sciences, 1975; 18-99.

28. JD Aplin. Cellular biochemistry of the endometrium. In: Wynn RM, Jollie WP (eds.), Biology of the Uterus. 2nd ed. New York: Plenum Publishers, 1989; 89-129.

29. AM Clark, MD Griswold. Expression of clusterin: sulfated glycoprotein-2 under conditions of heat stress in rat Sertoli cells and a mouse Sertoli cell line. J. Androl 1997; 18 257-263.

30. LE French, Sappino AP, Tschopp J, Schifferli JA. Human clusterin gene expression is confined to surviving cells during in vitro programmed cell death. J. Clin. Invest 1994; 93: 877-884. 